\title{
Identificação de perfis de comportamento do usuário para edificações residenciais multifamiliares e naturalmente ventiladas em Florianópolis
}

\author{
Identifying occupant behaviour profiles for multifamily \\ residential buildings with natural ventilation in \\ Florianópolis
}

\begin{abstract}
Bruna Faitão Balvedi Universidade Federal de Santa Catarina

Florianópolis - SC - Brasil

\section{Bruna Faitão Balvedi \\ Aline Schaefer \\ Mateus Vinícius Bavaresco \\ J oão Vítor Eccel \\ Enedir Ghisi}

\section{Resumo}

1

s interações entre o usuário e o ambiente construído compõem o comportamento do usuário. Esse conjunto de informações é essencial para aprimorar os resultados obtidos por meio de simulações computacionais, uma vez que o comportamento do usuário influencia o desempenho térmico e energético da edificação. Neste estudo, a influência do comportamento do usuário no desempenho térmico da edificação foi analisada a partir de simulações computacionais complementadas com dados de ocupação, operação de janelas e de persianas. Os perfis de comportamento do usuário foram identificados com a análise de agrupamento aplicada a uma base de dados monitorados em edificações residenciais multifamiliares de Florianópolis com ventilação natural. Os diferentes comportamentos do usuário foram responsáveis por variações superiores a 130\% nos resultados de graus hora de resfriamento e graus hora de aquecimento. De modo geral, a ocupação foi o aspecto do comportamento do usuário com maior influência no desempenho térmico devido ao aumento da carga térmica interna e a sua associação com a operação dos elementos da edificação, marcando uma dependência temporal (rotina).

Aline Schaefer

Palavras-chaves: Comportamento do usuário. Agrupamento. Desempenho térmico de edificações. Residencial. Simulação computacional.

Universidade Federal de Santa Catarina

Florianópolis - SC - Brasil

Mateus Vinícius Bavaresco Universidade Federal de Santa Catarina

Florianópolis - SC - Brasil

J oão Vítor Eccel

Abstract

Interactions between occupant and built environment define the occupant behaviour. This set of information is essential for improving results obtained from computer simulations, since occupant behaviour influences building thermal and energetic performance. In this study, the influence of the occupant behaviour on the thermal performance of a building was analysed through computer simulations implemented with data of occupancy, window and blind control. Occupant behaviour profiles were identified by means of cluster analysis based on data obtained from monitoring naturally-ventilated multifamily residential buildings in Florianópolis. Different occupant behaviours were responsible for up to $130 \%$ of variations on results of degree hours for cooling and degree hours for heating. In general, occupancy was the aspect of occupant behaviour with more influence on the thermal performance due to the increase in internal thermal load and its association with control of building elements, determining a time dependence (routine). Catarina

Recebido em 20/11/17 Aceito em 14/03/18

Keywords: Occupant behaviour. Clustering. Building thermal performance. Residential. Computer simulation.
\end{abstract}

BALVEDI, B. F.; SCHAEFER, A.; BAVARESCO, M. V.; ECCEL, J. V.; GHISI, E. Identificação de perfis de comportamento 


\section{Introdução}

As simulações computacionais são ferramentas fundamentais para estudar o desempenho térmico e energético de edificações. A partir dos resultados preditos torna-se possível otimizar a envoltória da edificação e seus sistemas com o intuito de promover conforto ambiental e reduzir o consumo de energia.

No Brasil, diversos estudos empregaram simulações com o programa computacional EnergyPlus (ENERGYPLUS, 2017) para estimar o desempenho térmico e energético de edificações residenciais. Fonseca et al. (2017) realizaram modelagem paramétrica para encontrar a melhor forma de uma edificação residencial levando em consideração os critérios de desempenho térmico e custo dos materiais construtivos. Ferreira, Souza e Assis (2017) avaliaram a influência dos limites das propriedades térmicas de diferentes normas nacionais no desempenho de edificações residenciais multifamiliares em 24 cidades brasileiras. Silva, Almeida e Ghisi (2017) avaliaram as incertezas quanto às propriedades térmicas e físicas dos materiais das edificações nas simulações computacionais e a influência disso na predição de desempenho térmico das edificações.

Apesar de o RTQ-R (INSTITUTO..., 2012) apresentar valores fixos para variáveis relacionadas ao controle da edificação no método de avaliação por simulação computacional, para que os resultados preditos aproximem-se da realidade de uso da edificação é essencial a utilização de dados referentes ao comportamento do usuário. Os dados de comportamento do usuário compreendem a ocupação dos ambientes e a operação dos elementos da edificação, tais como: janelas, persianas, sistemas de iluminação e sistemas de condicionamento artificial. Desse modo, o comportamento do usuário é composto pelas interações entre usuário e o ambiente construído e exerce influência direta sobre o desempenho termoenergético da edificação. De acordo com Andersen, Fabi e Corgnati (2016), uma predição realista do desempenho de uma edificação deve ser obtida por meio da inclusão de modelos realistas das interações do usuário com os controles da edificação. Estudos sobre a influência do comportamento do usuário no desempenho da edificação foram conduzidos por diversos autores que abordaram métodos para quantificar o impacto da variável usuário. A exemplo disso, a influência do usuário no consumo de energia foi estudada por Karjalainen (2016) por meio de simulações numéricas para um ambiente de escritório variando perfis de usuário: displicente, normal e consciente. Os diferentes perfis de usuário consideraram o consumo energético referente a equipamentos e sistema de iluminação. Os resultados apontaram que para um mesmo projeto e clima o usuário com perfil displicente pode consumir até $55 \%$ mais energia que o usuário consciente.

No estudo realizado por Taniguchi et al. (2016) o comportamento do usuário foi analisado quanto à sua influência sobre medidas de economia de energia. Além do comportamento do usuário, foram consideradas as seguintes características: composição familiar, área útil da residência, nível de isolamento. As simulações foram conduzidas com enfoque nos picos de demanda e foram implementadas com modelos de representação da distribuição de moradores e do estoque residencial em ampla escala. O comportamento com maior influência na redução da demanda de pico foi desligar as lâmpadas.

A diversidade de interações que compõem o comportamento do usuário agrega grande complexidade para os estudos de desempenho termoenergético, mesmo quando considerada uma única tipologia arquitetônica. Nesse sentido, a identificação de perfis contribui para que dados reais de comportamento do usuário possam ser implementados em simulações computacionais. A análise de agrupamento (análise de cluster) é uma técnica que permite analisar a diversidade de informações coletadas por meio do monitoramento do comportamento do usuário. Com esse tipo de análise, os dados monitorados são analisados e agrupados de acordo com a similaridade de suas características. Assim, cada grupo apresenta um perfil de comportamento semelhante internamente (alta homogeneidade interna) e perfis distintos entre grupos (alta heterogeneidade externa) (HAIR et al., 2009). Um dos resultados obtidos por meio dessa análise é a sintetização de informações para estudos em maior escala.

A análise de agrupamento foi utilizada por D’Oca e Hong (2015) para a obtenção de perfis típicos de ocupação em escritórios. Como variáveis foram utilizados fatores de conhecida influência sobre o comportamento, tais como: hora do dia, dia da semana e estado de ocupação anterior. No estudo de Aerts et al. (2014) a análise de agrupamento identificou sete perfis distintos para a ocupação de edificações residenciais. Na sequência, os perfis foram utilizados para calibração de um modelo estocástico desenvolvido pelos autores. A identificação de perfis de ocupação também foi conduzida por Duarte, van den Wymelenberg e Rieger (2013), o que resultou na criação de três grupos identificados pelos níveis baixo, médio e alto. 
Neste estudo, os perfis de comportamento do usuário, identificados por meio de análise de agrupamento, foram utilizados para avaliar a influência de diferentes usuários no desempenho térmico de quatro unidades habitacionais de uma mesma edificação multifamiliar de referência. Busca-se compreender melhor como a ocupação e as ações do usuário podem influenciar o desempenho térmico da edificação. A utilização de perfis de comportamento do usuário permite a aproximação dos dados de comportamento destes aos cenários reais de uso da edificação, favorecendo a predição dessas interações. Esse aspecto interativo é de fundamental importância ao projeto de novas edificações.

O objetivo geral deste trabalho é identificar perfis de comportamento do usuário para edificações multifamiliares naturalmente ventiladas e verificar sua influência no desempenho térmico de uma edificação multifamiliar de referência na cidade de Florianópolis, SC, por meio de análise de agrupamento e simulações computacionais.

\section{Método}

Para alcançar os objetivos deste trabalho, o método foi elaborado em três etapas:

(a) monitoramento do comportamento do usuário;

(b) análise de agrupamento; e

(c) simulações computacionais.

\section{Monitoramento do comportamento do usuário}

O desenvolvimento de uma base de dados sobre comportamento do usuário, incluindo ocupação, operação de janelas e operação de persianas, foi conduzido a partir da aplicação de questionários semiestruturados a moradores de edificações residenciais multifamiliares de Florianópolis, SC. Com base no modelo disponível em International Energy Agency - Energy in Buildings and Communities Annex 66 (INTERNATIONAL..., 2017), o questionário utilizado foi elaborado para obtenção de dados anuais sobre o comportamento do usuário.

Para cada unidade habitacional, um morador foi convidado a responder 15 questões, considerando apenas os ambientes de longa permanência - sala e dormitório. O comportamento referente à ocupação foi levantado com a distinção de dois períodos dias úteis e finais de semana - em questões nas quais o morador preenchia os horários em que permanecia em cada ambiente da unidade habitacional. $\mathrm{O}$ questionário abordou o comportamento do usuário em base anual, sem distinção entre período quente e frio, por exemplo (GHISI; BALVEDI, 2017). Tal representação foi feita em função dos resultados obtidos no estudo piloto, em que os participantes revelaram não haver ou não recordar diferenças no comportamento conforme as estações do ano. O estudo piloto foi elaborado para testar a formulação das questões, em especial na compreensão dos termos utilizados a pessoas não familiarizadas com a área de pesquisa (GHISI; BALVEDI, 2017).

A operação de janelas e de persianas foi levantada a partir de questões de múltipla escolha, que apresentavam como alternativas as variações do período de abertura de cada elemento. Dessa forma, buscou-se sintetizar a interação com os elementos de janelas e persianas por meio da indicação do tempo de abertura, variando de sempre aberta a sempre fechada. O tipo de sistema de condicionamento artificial também foi abordado no questionário. Entretanto, para este estudo, foram selecionados somente os casos em que os ambientes de longa permanência eram mantidos naturalmente ventilados.

A localização da edificação (bairro) e a altura do pavimento da unidade habitacional foram levantadas por meio do questionário. Não foram coletados dados socioeconômicos dos respondentes.

A aplicação do questionário ocorreu mediante a divulgação da sua versão digital na plataforma Formulários Google. A aplicação do questionário por meio digital permitiu a participação de público diverso, mantendo o pré-requisito de o respondente ser morador de edificação residencial multifamiliar de Florianópolis.

Os questionários recebidos foram verificados quanto a inconsistências e à totalidade nas respostas. Desse modo, apenas questionários completos foram considerados válidos.

Assim como outros métodos de monitoramento do comportamento do usuário, a aplicação de questionários acarreta incertezas nos dados, em função da subjetividade associada à própria resposta do usuário. Contudo, por se tratar de edificações residenciais, o método utilizado garante privacidade ao usuário e não interfere em suas atividades cotidianas, em oposição a métodos de monitoramento com equipamentos. A aplicação de questionários foi conduzida com sucesso por outros estudos direcionados a edificações residenciais (RICHARDSON; THOMSON; INFIELD, 2008; CHIOU et al., 2011; PINO; DE HERDE, 2011; CHEN et al., 2015; FENG et al., 2015). 


\section{Análise de agrupamento}

O processo de identificação de padrões conduzido pela análise de agrupamento baseia-se em medidas de homogeneidade entre os objetos e de heterogeneidade entre grupos de objetos (HAIR et al., 2009). Em função disso, essa ferramenta é capaz de identificar perfis de comportamento do usuário em uma amostra. A análise de agrupamento é processada em três etapas: tratamento das variáveis, cálculo da distância entre os objetos (que calcula o valor numérico do quão similares ou diferentes são dois objetos) e aplicação das técnicas de partição (que divide a amostra em subgrupos com base no grau de similaridade encontrado na etapa anterior). Durante o processo, foram adotados algoritmos de partição e medidas de similaridade em conformidade com o apresentado por Schaefer e Ghisi (2016). A aplicação da análise de agrupamento foi realizada com auxílio do programa Minitab 17 (MINITAB INC., 2013).

O tratamento das variáveis consiste em padronizálas de forma a evitar que a maior dispersão de determinadas variáveis influencie os resultados. A padronização estatística (z-scores) foi adotada para esse fim, visto que por meio dela obtém-se para cada variável um grupo de dados cuja média é igual a zero e desvio padrão igual a um. A Equação 1 apresenta a padronização estatística realizada.

$Z_{x_{i}}=\frac{\left(x_{i}-\bar{x}\right)}{s}$

Onde:

$Z_{x_{i}}$ é o valor padronizado de $x_{i}$;

$x_{i}$ é o valor que se deseja padronizar;

$\bar{x}$ é a média dos valores de determinada variável; e

s é o desvio padrão dos valores de determinada variável.

Na sequência, utilizou-se a Equação 2 para obter a distância entre cada par de objetos da amostra. A medida de similaridade utilizada foi a distância euclidiana quadrada.

$d_{A B}=\sum_{i=1}^{p}\left(x_{i_{A}}-x_{i_{B}}\right)^{2}$

Onde:

$d_{A B}$ é a distância euclidiana quadrada do objeto $\mathrm{A}$ ao objeto B;

$x_{i_{A}}$ é o valor de A para cada variável; e

$x_{i_{B}}$ é o valor de B para cada variável.

A partir das distâncias entre objetos, realizou-se o processo de partição, que consiste em distribuir os objetos de acordo com a semelhança ou não de suas características. Desse modo, perfis semelhantes de comportamento do usuário são agrupados em um mesmo grupo, enquanto perfis distintos são separados em grupos diferentes. A técnica hierárquica de partição juntamente com o Método de Ward (HAIR et al., 2009) foram selecionados para essa etapa da análise de agrupamento. O Método de Ward é um algoritmo de partição que separa os grupos de objetos com base na soma dos quadrados residuais dentro de cada grupo. A técnica hierárquica de partição analisa dois objetos a cada nova etapa e esse processo dá origem à construção de um dendograma, no qual são apresentados no eixo horizontal todos os objetos envolvidos na análise e, no eixo vertical, o grau de similaridade obtido a cada nova junção. Uma análise visual do dendograma permite identificar a formação dos diferentes grupos de uma amostra. A determinação do número ideal de grupos é feita pela análise dos níveis de similaridade a cada nova junção. Uma linha de corte é definida no dendograma quando a junção de objetos atinge um nível de similaridade relativamente superior aos anteriores.

Após a definição dos grupos mediante a análise de agrupamento, foram definidos objetos representativos de cada agrupamento. A definição dos objetos representativos baseou-se na distância entre objeto e o centroide de seu respectivo grupo. Desse modo, assume-se que as características apresentadas por esses perfis individuais representam características comuns ao comportamento do usuário de cada grupo. Assim, a inserção das características de cada objeto representativo nas simulações computacionais representará os diferentes perfis de comportamento do usuário encontrados para edificações multifamiliares de Florianópolis.

\section{Simulações computacionais}

As simulações de desempenho térmico da edificação de referência foram conduzidas com o programa computacional EnergyPlus (versão 8.8.0) e o arquivo climático INMET (LABORATÓRIO..., 2018), com extensão .epw, para Florianópolis, SC.

A edificação de referência utilizada foi desenvolvida por Montes (2016) a partir de dados nacionais de projetos arquitetônicos de edificações de interesse social destinadas à segunda faixa de renda familiar do Programa Minha Casa Minha Vida (BRASIL, 2016). A seleção desse modelo de edificação para a etapa de simulações computacionais reflete um objeto de estudo em comum: edificações residenciais multifamiliares. Assim, a similaridade entre as edificações é dada pela sua função - residencial - e pela sua principal característica projetual - multifamiliar. O projeto utilizado é composto de quatro unidades habitacionais distribuídas em pavimento tipo com 
formato em $\mathrm{H}$. As unidades habitacionais possuem mesma área e distribuição dos ambientes, diferenciando-se pela orientação solar. A planta baixa do pavimento tipo da edificação de referência utilizada é apresentada na Figura 1. A mesma nomenclatura das unidades habitacionais foi utilizada para identificação dos resultados simulados.

A Figura 2 apresenta a planta baixa da unidade habitacional (apartamento 1) com a identificação dos ambientes.

Além do projeto arquitetônico, também foram adotadas as características termofísicas dos elementos da envoltória obtidas por Montes (2016). A Tabela 1 apresenta os valores adotados para as características termofísicas de paredes e vidros.
Com o intuito de representar um pavimento tipo com altura intermediária, as superfícies de piso e cobertura foram mantidas adiabáticas. A edificação de referência foi considerada isolada no terreno para as simulações computacionais. Desse modo, a influência do entorno foi desconsiderada neste estudo.

As taxas metabólicas e potências instaladas de iluminação e equipamentos foram configuradas de acordo com o método de simulação do RTQ-R (INSTITUTO..., 2012), cujos valores estão apresentados na Tabela 2.

Dessa forma, os modelos simulados diferem somente quanto aos dados de comportamento do usuário referentes à ocupação, operação de janelas e operação de persianas.

Figura 1 - Planta baixa do pavimento tipo da edificação residencial multifamiliar de referência, desenvolvida por Montes (2016)

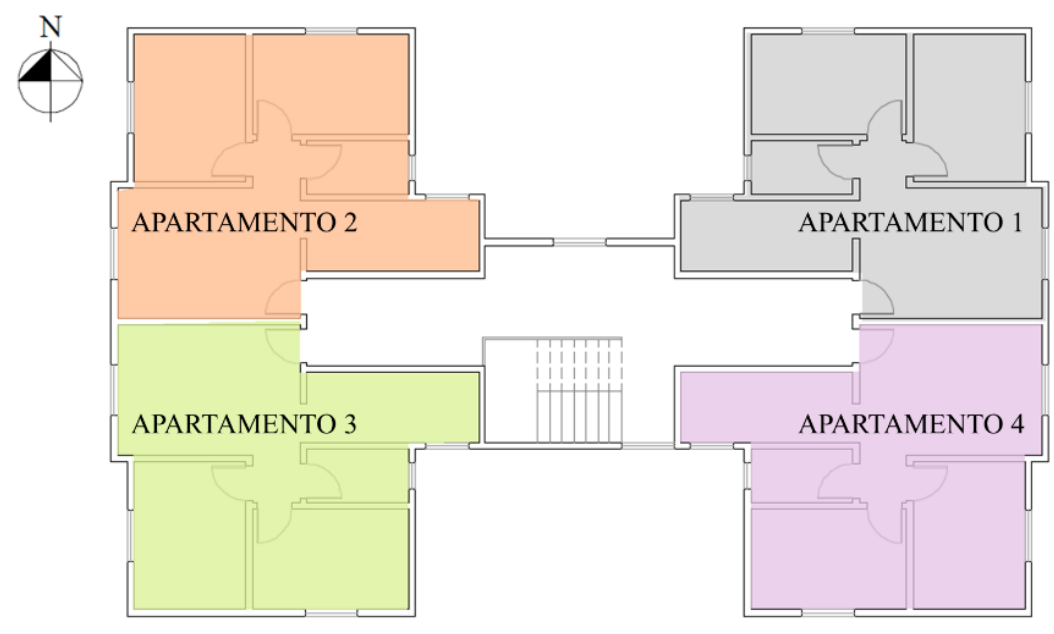

Figura 2 - Planta baixa da unidade habitacional - Apartamento 1

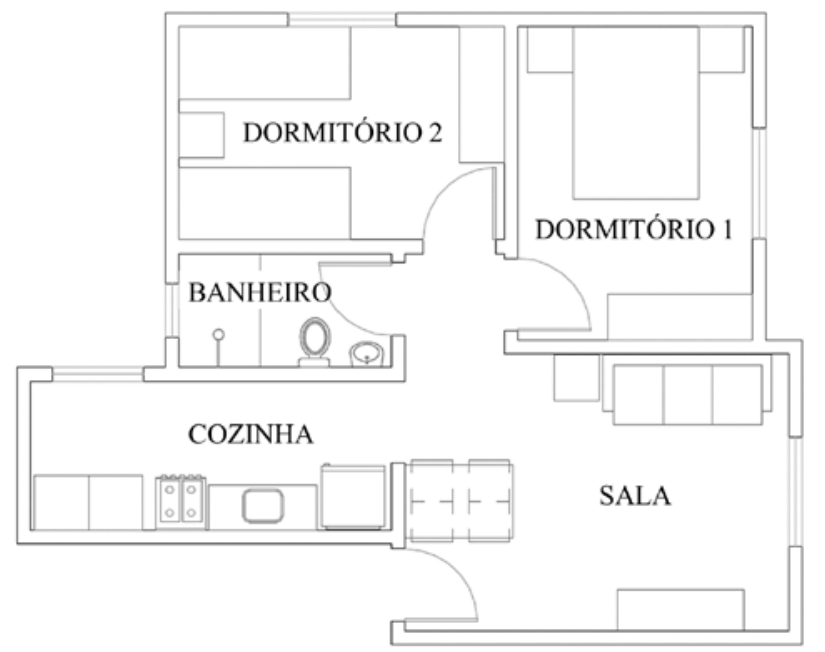

Fonte: Montes (2016). 
Tabela 1 - Propriedades termofísicas dos elementos da envoltória

\begin{tabular}{c|c|c|c|c}
\hline $\begin{array}{c}\text { Transmitância } \\
\text { térmica da parede } \\
{\left[\mathbf{W} /\left(\mathbf{m}^{\mathbf{2}} \mathbf{K}\right)\right]}\end{array}$ & $\begin{array}{c}\text { Capacidade } \\
\text { térmica da parede } \\
{\left[\mathbf{k J} / \mathbf{m}^{\mathbf{2}} \mathbf{K}\right]}\end{array}$ & $\begin{array}{c}\text { Absortância } \\
\text { solar da parede }\end{array}$ & $\begin{array}{c}\text { Transmitância } \\
\text { térmica do vidro } \\
{\left[\mathbf{W} /\left(\mathbf{m}^{2} \mathbf{K}\right)\right]}\end{array}$ & $\begin{array}{c}\text { Fator solar do } \\
\text { vidro }\end{array}$ \\
\hline 2,78 & 209 & 0,7 & 5,8 & 0,8 \\
\hline
\end{tabular}

Tabela 2 - Cargas térmicas internas configuradas para cada zona térmica da edificação simulada

\begin{tabular}{l|c|c|c}
\hline Zona térmica & $\begin{array}{c}\text { Taxa } \\
\text { metabólica } \\
{\left[\mathbf{W} / \mathbf{m}^{2}\right]}\end{array}$ & $\begin{array}{c}\text { Potência instalada } \\
\text { de iluminação } \\
{\left[\mathbf{W} / \mathbf{m}^{2}\right]}\end{array}$ & $\begin{array}{c}\text { Potência } \\
\text { instalada de } \\
\text { equipamentos } \\
{\left[\mathbf{W} / \mathbf{m}^{2}\right]}\end{array}$ \\
\hline Dormitório 1 & 45 & 5,0 & - \\
Dormitório 2 & 45 & 5,0 & - \\
Sala/cozinha integradas & 60 & 6,0 & 1,5 \\
\hline
\end{tabular}

A análise dos resultados obtidos por meio das simulações computacionais foi conduzida utilizando as temperaturas operativas de cada zona térmica e os indicadores de desempenho térmico graus hora de aquecimento e de resfriamento. Esses indicadores são obtidos pelo somatório da diferença entre a temperatura operativa do ambiente de longa permanência e limites pré-fixados de conforto térmico. Os limites de $18{ }^{\circ} \mathrm{C}$ e $26{ }^{\circ} \mathrm{C}$ foram utilizados para determinar os graus hora de aquecimento e de resfriamento, respectivamente. Os indicadores de graus hora são frequentemente associados a análises de desempenho térmico de edificações naturalmente ventiladas, uma vez que consideram condições de conforto térmico sem o uso de condicionamento artificial (VERSAGE, 2009).

A análise da influência do comportamento do usuário no desempenho térmico foi conduzida mediante comparação dos resultados de graus hora de aquecimento e de resfriamento para cada ambiente de longa permanência. A comparação dos resultados obtidos para os diferentes perfis de comportamento do usuário foi utilizada para identificar a influência que variações de ocupação, operação de janelas e operação de persianas geram sobre o desempenho térmico das zonas térmicas.

Os resultados obtidos para as quatro unidades habitacionais também foram comparados com o intuito de identificar a influência da orientação solar sobre o desempenho térmico das zonas estudadas. A combinação dos efeitos gerados pela variação do comportamento do usuário e da orientação solar contribui para o estudo do desempenho térmico da edificação ao aproximar-se de cenários reais de uso.

\section{Resultados}

\section{Monitoramento do comportamento do usuário}

A aplicação de questionários a moradores de edificações residenciais multifamiliares de Florianópolis, durante o período de três meses, resultou em uma base de dados de 100 respostas válidas. A partir dessa base de dados, foram selecionados os casos referentes a unidades habitacionais cujos ambientes de longa permanência são mantidos exclusivamente com ventilação natural, isto é, sem a presença de sistema de condicionamento artificial. Portanto, a base efetiva a ser utilizada nas etapas seguintes é composta de 25 casos.

\section{Análise de agrupamento}

A análise de agrupamento conduzida mediante a técnica hierárquica de partição resultou na construção de um dendograma. Neste estudo, cada objeto representa os dados de comportamento do usuário fornecidos por um morador de edificações multifamiliares de Florianópolis. A linha de corte para a definição do número ideal de grupos considerou o nível de similaridade obtido na terceira junção e sua superioridade em relação aos níveis anteriores. Conforme apresentado na Figura 3, o dendograma aponta para a presença de três perfis distintos.

Os resultados da análise de agrupamento mostram que não há distribuição igual entre os perfis resultantes. Os perfis 2 e 3 são compostos de doze e nove objetos, respectivamente, enquanto o perfil 1 possui somente quatro objetos. Como a distribuição dos objetos durante a análise de agrupamento é realizada em função da medida de similaridade e do algoritmo de partição, uma concentração maior de objetos em determinado perfil não está relacionada 
ao tamanho da amostra, e sim às características dos objetos.

Para cada perfil de comportamento do usuário foi selecionado um objeto representativo, a partir da menor distância entre o objeto e o centroide. As características dos objetos representativos dos três perfis de comportamento do usuário foram inseridas nas simulações computacionais, conforme apresentadas no Quadro 1.

Figura 3 - Dendograma do processo de agrupamento dos perfis de ocupação e operação, obtido com o Minitab

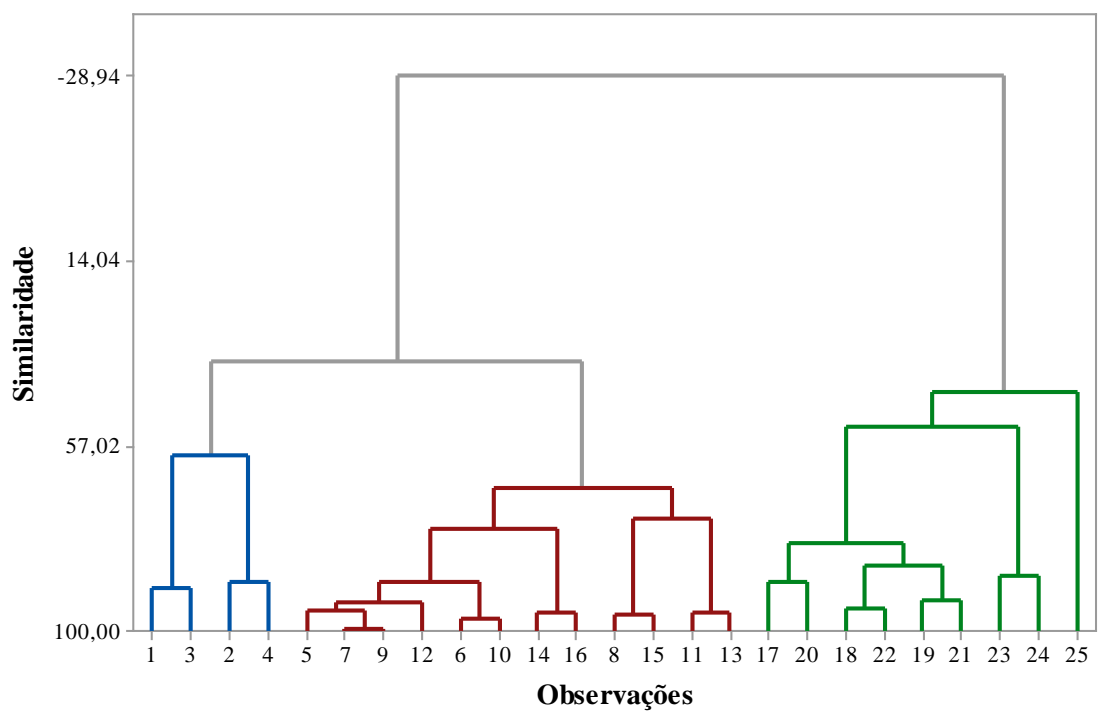

Quadro 1 - Características dos perfis avaliados de comportamento do usuário

\begin{tabular}{|c|c|c|c|c|}
\hline \multicolumn{2}{|c|}{ Características do comportamento } & Perfil 1 & Perfil 2 & Perfil 3 \\
\hline \multirow{2}{*}{$\begin{array}{l}\text { Ocupação da } \\
\text { sala/cozinha }\end{array}$} & Dias úteis & $\begin{array}{l}\text { Das 10h às } \\
\text { 23h59min }\end{array}$ & $\begin{array}{l}\text { Das } 22 \mathrm{~h} \text { à } \\
1 \mathrm{~h} 59 \mathrm{~min}\end{array}$ & $\begin{array}{l}\text { Das } 7 \mathrm{~h} \text { às } 7 \mathrm{~h} 59 \mathrm{~min} \text {, } \\
\text { das } 12 \mathrm{~h} \text { às } 12 \mathrm{~h} 59 \mathrm{~min} \\
\text { e das } 20 \mathrm{~h} \text { às } \\
21 \mathrm{~h} 59 \mathrm{~min}\end{array}$ \\
\hline & $\begin{array}{l}\text { Finais de } \\
\text { semana }\end{array}$ & $\begin{array}{l}\text { Das 10h às } \\
\text { 23h59min }\end{array}$ & $\begin{array}{l}\text { Das } 15 \mathrm{~h} \text { à } \\
\text { 0h59min }\end{array}$ & $\begin{array}{l}\text { Das } 11 \mathrm{~h} \text { às } \\
12 \mathrm{~h} 59 \mathrm{~min} \text { e das } 17 \mathrm{~h} \\
\text { às } 20 \mathrm{~h} 59 \mathrm{~min} \\
\end{array}$ \\
\hline \multirow[b]{2}{*}{$\begin{array}{l}\text { Ocupação dos } \\
\text { dormitórios }\end{array}$} & Dias úteis & De 0h a 8h59min & Das 2h às 8h59min & Das 21h às 6h59min \\
\hline & $\begin{array}{l}\text { Finais de } \\
\text { semana }\end{array}$ & De $0 \mathrm{~h}$ a $8 \mathrm{~h} 59 \mathrm{~min}$ & $\begin{array}{l}\text { Da 1h às } \\
\text { 10h59min }\end{array}$ & $\begin{array}{l}\text { Das 21h às } \\
\text { 10h59min }\end{array}$ \\
\hline \multirow{2}{*}{$\begin{array}{l}\text { Operação das } \\
\text { cortinas/persianas }\end{array}$} & Sala & Sempre fechada & $\begin{array}{l}\text { Aberta quando o } \\
\text { ambiente é } \\
\text { ocupado }\end{array}$ & Sempre aberta \\
\hline & Dormitórios & Sempre fechada & $\begin{array}{l}\text { Aberta quando as } \\
\text { janelas estão } \\
\text { abertas }\end{array}$ & Sempre aberta \\
\hline \multirow{2}{*}{$\begin{array}{l}\text { Operação das } \\
\text { janelas }\end{array}$} & Sala & $\begin{array}{l}\text { Aberta quando o } \\
\text { ambiente é } \\
\text { ocupado }\end{array}$ & $\begin{array}{l}\text { Aberta quando o } \\
\text { ambiente é } \\
\text { ocupado }\end{array}$ & $\begin{array}{l}\text { Aberta quando o } \\
\text { ambiente é ocupado }\end{array}$ \\
\hline & Dormitórios & Sempre fechada & $\begin{array}{l}\text { Aberta por um } \\
\text { curto intervalo } \\
\text { diário para renovar } \\
\text { o ar }\end{array}$ & $\begin{array}{l}\text { Aberta quando o } \\
\text { ambiente é ocupado }\end{array}$ \\
\hline
\end{tabular}


Para os casos em que a operação das janelas foi descrita como “Aberta por um curto intervalo diário para renovar o ar" optou-se por simular a abertura das janelas durante 30 minutos no início e no final de cada período de ocupação. As portas internas foram mantidas sempre abertas em todos os perfis simulados.

Os perfis diferem bastante quanto à ocupação dos ambientes de longa permanência, variando, por exemplo, de poucas horas, não contínuas, de ocupação na sala em dias úteis para o perfil 3 para até 14 horas de ocupação contínua para o perfil 1. A variação quanto à operação de persianas aponta para dois extremos do comportamento do usuário, sendo mantidas sempre fechadas no perfil 1 e sempre abertas no perfil 3. Observa-se uma relação entre a operação de janelas e a ocupação, nas salas, para todos os perfis. Contudo, as características da ocupação são bastante distintas, o que faz com que a disponibilidade de ventilação natural também se diferencie para cada um dos perfis de comportamento do usuário.

Os perfis representativos de comportamento do usuário foram inseridos nas simulações computacionais de forma estática, ou seja, foram utilizados horários fixos de ocupação e de interação com janelas e persianas, com distinção apenas para dias úteis e finais de semana. Esses perfis correspondem às informações apresentadas no Quadro 1 e têm origem no monitoramento de dados desenvolvido com moradores de edificações multifamiliares de Florianópolis.

\section{Simulações computacionais}

As simulações computacionais realizadas com o programa EnergyPlus resultaram em dados horários de temperatura operativa, os quais foram avaliados mediante o indicador de desempenho térmico graus hora. A Figura 4 apresenta os resultados obtidos para os graus hora de resfriamento dos ambientes de longa permanência das quatro unidades habitacionais que compõem o pavimento tipo da edificação multifamiliar de referência. Os três perfis apontados correspondem à variação do comportamento do usuário a partir dos dados de ocupação, operação de janelas e operação de persianas.

Os resultados de graus hora de aquecimento relacionados aos ambientes de longa permanência das quatro unidades habitacionais são apresentados na Figura 5.

A comparação dos resultados aponta para maiores valores de graus hora para resfriamento do que para aquecimento, para todos os ambientes e perfis de comportamento do usuário. Isso reflete a influência combinada entre características do clima e da envoltória, somadas às cargas internas devidas ao comportamento do usuário.

Os valores obtidos para graus hora de resfriamento demonstram a proximidade dos resultados dos perfis 1 e 2 e sua distinção em relação ao perfil 3 . Em contrapartida, tal tendência não é verificada nos resultados de graus hora de aquecimento.

\section{Figura 4 - Graus hora de resfriamento para os ambientes de longa permanência das quatro unidades habitacionais do pavimento tipo da edificação multifamiliar}

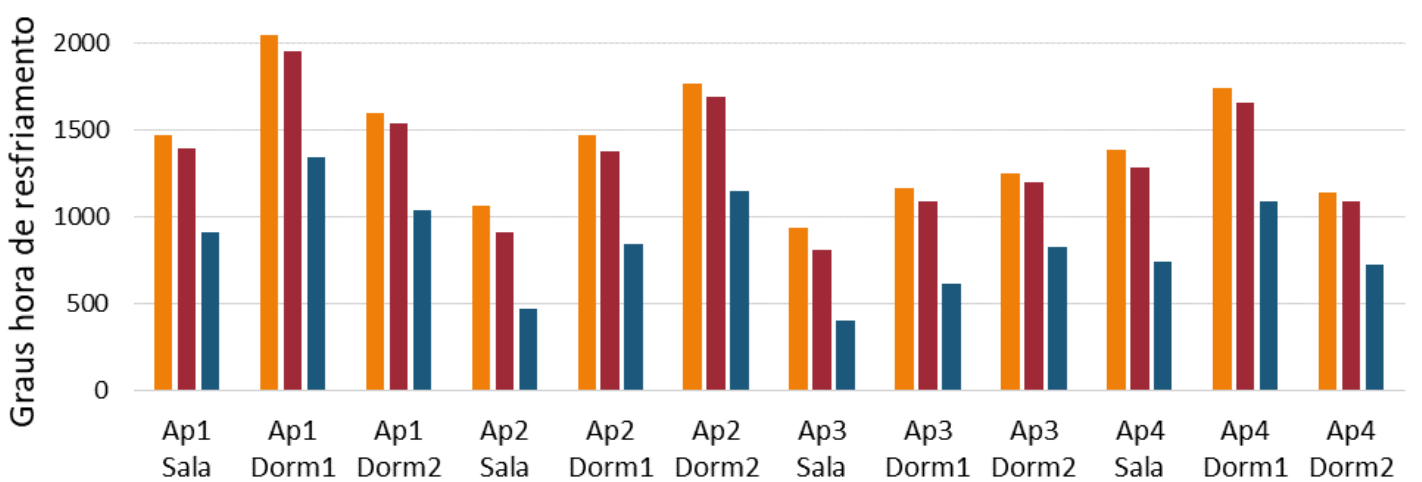

- Perfil 1 Perfil $2 \square$ Perfil 3 


\section{Figura 5 - Graus hora de aquecimento para os ambientes de longa permanência das quatro unidades} habitacionais do pavimento tipo da edificação multifamiliar

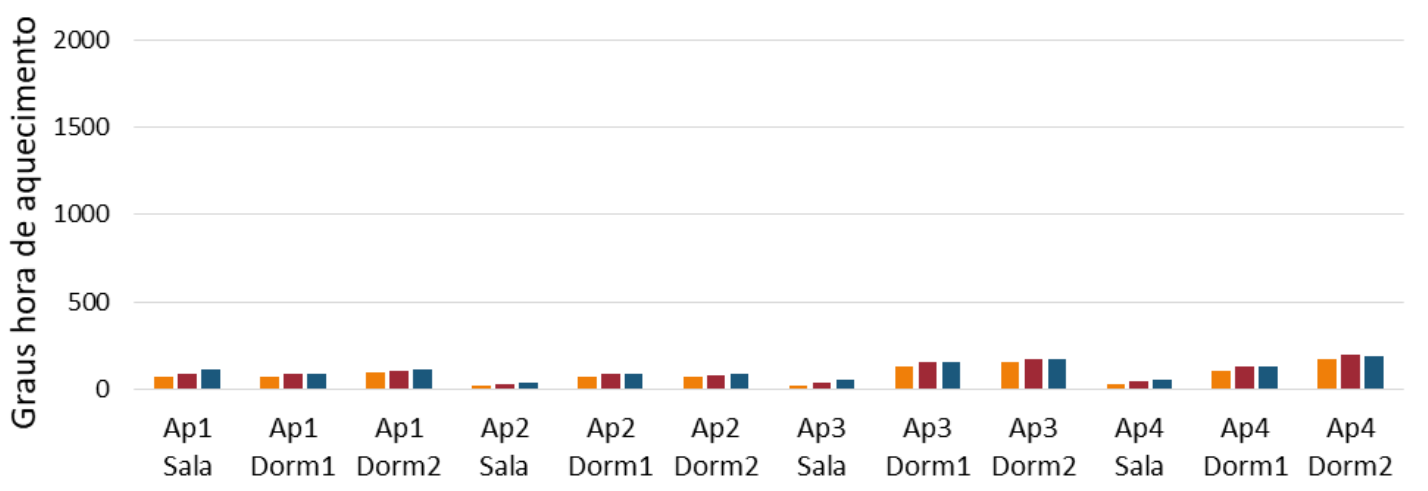

- Perfil 1 - Perfil 2 - Perfil 3

A distinção entre as características de ocupação, operação de janela e de persiana foi o fator considerado pela análise de agrupamento para a definição de três perfis de comportamento do usuário. Portanto, a proximidade dos resultados de graus hora de resfriamento dos perfis 1 e 2 reflete as consequências das ações desses usuários, sendo que uma combinação de diferentes ações pode acarretar resultados absolutos próximos. Por exemplo, enquanto o usuário do perfil 1 mantém as persianas sempre fechadas na sala, o usuário do perfil 2 as mantém abertas durante a ocupação. Entretanto, há uma diferença de 10 horas entre o período de ocupação desse mesmo ambiente durante os dias úteis, para os dois perfis.

O comportamento do usuário do perfil 3 resultou em menor graus hora de resfriamento para todos os ambientes. Em contrapartida, o perfil 3 obteve maiores valores de graus hora de aquecimento. $\mathrm{O}$ principal diferencial do comportamento do usuário do perfil 3 refere-se à operação das janelas, as quais são mantidas abertas durante a ocupação dos ambientes de longa permanência. Para os dormitórios, por exemplo, isso representa uma estratégia de ventilação noturna, a qual contribui para a perda de calor em função de temperaturas externas mais amenas durante esse período. Em vista de os perfis de comportamento do usuário não diferenciarem estações do ano, a estratégia de ventilação noturna durante o período de inverno contribuiu para que os dormitórios do perfil 3 apresentassem maiores valores de graus hora de aquecimento.

A manutenção de janelas e persianas fechadas por mais tempo, conforme visto no comportamento do usuário dos perfis1 e 2, gerou maior graus hora de resfriamento para todos os ambientes, em comparação com os observados para o perfil 3.

O perfil 1 apresenta a maior quantidade de horas de ocupação na sala, em comparação com os demais perfis, o que reflete em aumento da carga térmica interna. Além disso, a ocupação da sala no perfil 1 ocorre durante todo o período vespertino, caracterizado por temperaturas do ar externo mais elevadas. Como resultado, o perfil 1 apresenta os maiores valores de graus hora de resfriamento.

A maior diferença entre os graus hora de resfriamento refere-se à sala do apartamento 3. Para esse ambiente, o resultado de graus hora de resfriamento do perfil 1 foi $131 \%$ maior do que o encontrado para o perfil 3. Quanto aos graus hora de aquecimento, a maior diferença foi encontrada para a sala do apartamento 4, para o qual o resultado do comportamento do perfil 3 foi $132 \%$ superior ao do perfil 1.

Os dormitórios com abertura orientada a sul (dormitório 2, nos apartamentos 3 e 4) obtiveram os maiores valores de graus hora de aquecimento. Esse resultado foi observado para os três perfis de comportamento do usuário. Os dormitórios com maiores valores de graus hora de resfriamento possuem abertura orientada a leste (dormitório 1, nos apartamentos 1 e 4) e a norte (dormitório 2, nos apartamentos 1 e 2).

Montes (2016) avaliou o desempenho térmico da edificação de referência considerando o método prescritivo do Regulamento Técnico da Qualidade para o Nível de Eficiência Energética de Edificações Residenciais - RTQ-R (BRASIL, 2012) do Inmetro/Procel Edifica, para a zona bioclimática 3. A edificação com pavimento tipo em formato $\mathrm{H}$ corresponde à renda 2 do Programa Minha Casa Minha Vida (BRASIL, 2016) e teve uma unidade habitacional (apartamento 1) avaliada. Os resultados obtidos pela autora revelam que apesar de atender aos requisitos mínimos referentes às paredes e à ventilação nas aberturas dos dormitórios, a unidade habitacional não apresenta desempenho térmico satisfatório. Foi obtido nível E para a envoltória, considerando a unidade 
habitacional em pavimento tipo. Quanto ao resfriamento para verão, foram obtidos níveis $\mathrm{D}$ para os dormitórios (1 e 2) e nível E para a sala.

A edificação de referência utilizada nas simulações representa projetos arquitetônicos comumente reproduzidos para habitações de interesse social. Em função disso, os ambientes de longa permanência possuem dimensões reduzidas que evidenciam a influência do comportamento do usuário. A ocupação e as operações de janela e persiana apresentam efeito direto no aumento da carga térmica interna e no ganho de calor, o que pôde ser observado nos indicadores de desempenho térmico.

Conforme visto, a operação dos elementos da edificação foi associada à ocupação, o que sugere que o usuário esteja priorizando outros fatores em detrimento do conforto térmico, tais como: rotina, privacidade, conforto visual, vista para o exterior. Nesse sentido, pondera-se que simulações computacionais que vinculam a operação de janelas e persianas exclusivamente à temperatura externa deixam de abordar outros aspectos que são inerentes a cenários reais de operação dos elementos da edificação.

\section{Conclusões}

Este trabalho desenvolveu uma base de dados com informações sobre o comportamento do usuário em edificações residenciais multifamiliares de Florianópolis, a partir da qual foram selecionados 25 casos correspondentes às unidades habitacionais naturalmente ventiladas. Por meio de análise de agrupamento utilizando técnica hierárquica de partição foram identificados três perfis de comportamento do usuário dentro da amostra de dados. Um objeto representativo de cada grupo foi selecionado e suas informações inseridas como dados de entrada nas simulações computacionais com o programa EnergyPlus para o pavimento tipo de uma edificação multifamiliar de referência. A influência da variação do comportamento do usuário no desempenho térmico da edificação foi analisada mediante os indicadores de graus hora. Os resultados apontaram para a significativa influência da operação das janelas na manutenção de condições internas dentro dos limites de conforto térmico. A influência dessa ação do usuário foi evidenciada na comparação dos resultados obtidos para o perfil 3, no dormitório, para o qual se verificou a estratégia de ventilação noturna. A influência da ocupação foi observada nos resultados de graus hora de aquecimento, uma vez que a presença do usuário representa aumento da carga térmica interna. Tal efeito foi evidenciado pelos resultados obtidos para o perfil 1 de comportamento do usuário. O perfil 1 também demonstrou maior influência entre a combinação de comportamento do usuário e orientação solar, visto que se obteve variação de até $343 \%$ nos valores de graus hora de aquecimento e $125 \%$ nos valores de graus hora de resfriamento, para a sala das diferentes unidades habitacionais. Para os dormitórios, observou-se que a orientação solar da abertura foi um fator de influência, independentemente do comportamento do usuário. Pondera-se que, assim como a orientação solar, outros fatores que não foram foco deste trabalho podem apresentar influências significativas sobre o desempenho térmico de uma edificação. A análise dos resultados das simulações mostrou que apesar dos três perfis possuírem características diferentes de ocupação e operação de janelas e persianas, os resultados de graus hora podem ser semelhantes, conforme visto para os graus hora de resfriamento dos perfis 1 e 2 . As características projetuais da edificação de referência analisada contribuíram para evidenciar a influência do comportamento do usuário no desempenho térmico dos ambientes de longa permanência. Por exemplo, o aumento da carga térmica interna representada pela ocupação foi bastante significativo em função da reduzida área dos ambientes. Por fim, observou-se que em determinados perfis de comportamento do usuário a operação dos elementos da edificação foi associada ao período de ocupação. De modo geral, a ocupação dos ambientes de longa permanência pode ser destacada por sua influência sobre o desempenho térmico das unidades habitacionais simuladas, visto que além de afetar a carga térmica interna, a ocupação é condicional para determinadas ações, como, por exemplo, manter as janelas abertas quando o usuário está no ambiente. A soma desses fatores marca uma dependência temporal do comportamento do usuário, assinalada pela rotina e por vezes com maior influência que o conforto térmico. A utilização dos perfis de comportamento do usuário identificados neste estudo fica restrita a simulações conduzidas para o mesmo local e objeto de estudo. Tal restrição se deve ao fato de o comportamento do usuário ser intrinsecamente relacionado ao clima, à cultura local e à arquitetura.

\section{Referências}

AERTS, D. et al. A Method For the Identification and Modelling of Realistic Domestic Occupancy Sequences For Building Energy Demand Simulations and Peer Comparison. Building and Environment, v. 75, p. 67-78, 2014. 
ANDERSEN, R. K.; FABI, V.; CORGNATI, S. P. Predicted and Actual Indoor Environmental Quality: verification of occupants' behaviour models in residential buildings. Energy and Buildings, v. 127, p. 105-115, 2016.

BRASIL. Programa Minha Casa Minha Vida. Disponível em: $<$ http://www.minhacasaminhavida.gov.br/sobreo405programa.html>. Acesso em: 15 mar. 2016.

BRASIL. Regulamento Técnico da Qualidade para o Nível de Eficiência Energética Edificações Residenciais. Disponível em: $<$ http://www.inmetro.gov.br/legislacao/rtac/pdf/RT AC001788.pdf>. Acesso em: 15 mar. 2012.

CHEN, S. et al. Definition of Occupant Behavior in Residential Buildings and Its Application to Behavior Analysis in Case Studies. Energy and Buildings, v. 104, p. 1-13, 2015.

CHIOU, Y. S. et al. A high Spatial Resolution Residential Energy Model Based on American Time Use Survey Data and the Bootstrap Sampling Method. Energy and Buildings, v. 43, n. 12, p. 3.528-3.538, 2011.

D’OCA, S.; HONG, T. Occupancy Schedules Learning Process Through a Data Mining Framework. Energy and Buildings, v. 88, p. 395408, 2015.

DUARTE, C.; VAN DEN WYMELENBERG, K.; RIEGER, C. Revealing Occupancy Patterns in an Office Building Through the Use of Occupancy Sensor Data. Energy and Buildings, v. 67, p. 587 595, 2013.

ENERGYPLUS. Energy Simulation Software. Disponível em: <https://energyplus.net/>. Acesso em: 15 dez. 2017.

FENG, X. et al. A Preliminary Research on the Derivation of Typical Occupant Behavior Based on Large-Scale Questionnaire Surveys. Energy and Buildings, v. 117, p. 332-340, 2015.

FERREIRA, C. C.; SOUZA, H. A. de; ASSIS, E. S. de. Discussão dos Limites das Propriedades Térmicas dos Fechamentos Opacos Segundo as Normas de Desempenho Térmico Brasileiras.

Ambiente Construído, Porto Alegre, v. 17, n. 1, p. 183-200, jan./mar. 2017.

FONSECA, L. P. G. et al. Otimização Multiobjectivo das Dimensões dos Ambientes de Uma Residência Unifamiliar Baseada em Simulação Energética e Estrutural. Ambiente Construído, Porto Alegre, v. 17, n. 1, p. 267-288, jan./mar. 2017.
GHISI, E.; BALVEDI, B. F. Monitoring Occupant Behaviour in Multifamily Residential Buildings. In: PLEA, Edinburgh, 2017. Proceedings... Edinburgh, 2017.

HAIR, J. F. et al. Análise Multivariada de Dados. 6. ed. Porto Alegre: Bookman, 2009.

INSTITUTO NACIONAL DE METROLOGIA E QUALIDADE INDUSTRIAL. Regulamento

Técnico da Qualidade para o Nível de Eficiência Energética de Edificações Residenciais (RTQR). Rio de Janeiro: INMETRO, 2012. Portaria n. 18.

\section{INTERNATIONAL ENERGY AGENCY \\ ENERGY IN BUILDINGS AND \\ COMMUNITIES PROGRAM. Occupants}

Behavior Research Bibliography. Disponível em: <http:// www.annex66.org/?q=biblio>. Acesso em: 15 dez. 2017.

KARJALAINEN, S. Should We Design Buildings That Are Less Sensitive to Occupant Behaviour? A simulation study of effects of behaviour and design on office energy consumption. Energy Efficiency, p. 1-14, 2016.

\section{LABORATÓRIO DE EFICIÊNCIA \\ ENERGÉTICA EM EDIFICAÇÕES. Arquivos}

Climáticos. Disponível em:

$<$ http://www.labeee.ufsc.br/downloads/arquivosclimaticos>. Acesso em: 15 jan. 2018.

MINITAB INC. Minitab (Version 17.1.0.0), Software. 2013. Disponível em:

$<$ http://www.minitab.com>. Acesso em: 15 mar. 2016.

MONTES, M. A. T. Abordagem Integrada no Ciclo de Vida de Habitação de Interesse Social Considerando Mudanças Climáticas.

Florianópolis, 2016. Tese (Doutorado em Engenharia Civil) - Programa de Pós-Graduação em Engenharia Civil, Universidade Federal de Santa Catarina, Florianópolis, 2016.

PINO, F. E.; DE HERDE, A. Definition of Occupant Behaviour Patterns With Respect to Ventilation for Apartments From the Real Estate Market in Santiago de Chile. Sustainable Cities and Society, v. 1, n. 1, p. 38-44, 2011.

RICHARDSON, I.; THOMSON, M.; INFIELD, D. A High-Resolution Domestic Building Occupancy Model For Energy Demand Simulations. Energy and Buildings, v. 40, n. 8, p. 1.560-1.566, 2008.

SCHAEFER, A.; GHISI, E. Method For Obtaining Reference Buildings. Energy and Buildings, v. 128, p. 660-672, 2016. 
SILVA, A. S.; ALMEIDA, L. S. S.; GHISI, E.

Análise de Incertezas Físicas em Simulação

Computacional de Edificações Residenciais.

Ambiente Construído, Porto Alegre, v. 17, n. 1, p. 289-303, jan./mar. 2017.

TANIGUCHI, A. et al. Estimation of the Contribution of the Residential Sector to Summer Peak Demand Reduction in Japan Using an Energy End-Use Simulation Model. Energy and

Buildings, v. 112, p. 80-92, 2016.
VERSAGE, R. de S. Ventilação Natural e Desempenho Térmico de Edifícios Verticais Multifamiliares em Campo Grande, MS.

Florianópolis, 2009. Dissertação (Mestrado em Engenharia Civil) - Programa de Pós-graduação em Engenharia Civil, Universidade Federal de Santa Catarina, Florianópolis, 2009.

\section{Bruna Faitão Balvedi}

Departamento de Engenharia Civil, Centro Tecnológico, Laboratório de Eficiência Energética em Edificações | Universidade Federal de Santa Catarina | Trindade | Florianópolis - SC - Brasil | CEP 88040-900 | Tel.: (48) 3721-5184 | E-mail: brunabalvedi@live.com

\section{Aline Schaefer}

Departamento de Engenharia Civil, Centro Tecnológico, Laboratório de Eficiência Energética em Edificações | Universidade Federal de Santa Catarina | E-mail: alineschaefer.au@gmail.com

\section{Mateus Vinícius Bavaresco}

Departamento de Engenharia Civil, Centro Tecnológico, Laboratório de Eficiência Energética em Edificações | Universidade Federal de Santa Catarina | E-mail: bavarescomateus@gmail.com

\section{J oão Vítor Eccel}

Departamento de Engenharia Civil, Centro Tecnológico | Universidade Federal de Santa Catarina | E-mail: joao_eccel@hotmail.com

\section{Enedir Ghisi}

Departamento de Engenharia Civil, Centro Tecnológico, Laboratório de Eficiência Energética em Edificações | Universidade Federal de Santa Catarina | Tel.: (48) 3721-2115 | E-mail: enedir@labeee.ufsc.br

\section{Revista Ambiente Construído}

Associação Nacional de Tecnologia do Ambiente Construído

Av. Osvaldo Aranha, 99 - 3o andar, Centro

Porto Alegre - RS - Brasil

CEP $90035-190$

Telefone: +55 (51) 3308-4084

Fax: +55 (51) 3308-4054

www. seer. ufrgs. br/ ambienteconstruido

E-mail: ambienteconstruido@ufrgs.br 